\title{
Les effets du tramway sur la fréquentation du transport public. Un bilan des agglomérations françaises de province
}

Tram effects on public transport use. An assessment of French provincial urban areas

\section{Vincent Gagnière}

\section{(2) OpenEdition Journals}

Édition électronique

URL : http://journals.openedition.org/rge/3508

DOI : $10.4000 /$ rge.3508

ISSN : 2108-6478

Éditeur

Association des géographes de l'Est

Édition imprimée

Date de publication : 15 juin 2012

ISSN : 0035-3213

Référence électronique

Vincent Gagnière, «Les effets du tramway sur la fréquentation du transport public. Un bilan des agglomérations françaises de province », Revue Géographique de l'Est [En ligne], vol. 52 / 1-2 | 2012, mis en ligne le 16 octobre 2012, consulté le 10 décembre 2020. URL : http://journals.openedition.org/ rge/3508; DOI : https://doi.org/10.4000/rge.3508

Ce document a été généré automatiquement le 10 décembre 2020.

Tous droits réservés 


\title{
Les effets du tramway sur la fréquentation du transport public. Un bilan des agglomérations françaises de province
}

Tram effects on public transport use. An assessment of French provincial urban areas

\author{
Vincent Gagnière
}

\section{Introduction}

1 Vingt-cinq ans ont passé depuis les premières réintroductions de lignes de tramway dans les centres urbains de province, à Nantes en 1985 et à Grenoble en 1987. Depuis le milieu des années 1990, la multiplication des ouvertures offre un contraste saisissant avec les fermetures de lignes dans les années 1960. En trois décennies, le retournement de situation est total. Les collectivités locales investissent plusieurs centaines de millions d'euros pour rouvrir des lignes de tramway, parfois là même où les anciens réseaux ont été fermés trente ans plus tôt.

2 Face à une telle success story modale et à une telle dépense publique, la recherche multiplie les évaluations des effets de la réintroduction du tramway dans les centres urbains des agglomérations françaises de province. De nombreux angles d'analyse sont mobilisés, en province comme en Île-de-France, à la croisée des champs disciplinaires : l'économie permet de s'interroger sur le bilan carbone de telles politiques (Prud'homme, Koning et Kopp, 2011), comme sur leurs effets sur les prix immobiliers (Fritsch, 2007); alliée à la géographie, elle interroge les modifications d'accessibilité engendrées par ce mode de transport (Mercier, 2008); l'aménagement envisage les effets du tramway dans une approche urbaine globale (Stambouli, 2007), ou selon l'angle spécifique de la fréquentation des transports collectifs (Beaucire, 2006). 
3 C'est cette dimension que cet article se propose de mettre à jour, pour trois raisons. L'actualisation du bilan est d'abord justifiée par la multiplication des mises en service : depuis 2001, date de la plus récente mise en service analysée par Francis Beaucire, huit nouveaux réseaux viennent compléter le panel, le portant à 14 agglomérations, et permettant d'affiner les résultats. De plus, plusieurs avancées empiriques rendent nécessaire de creuser l'effet supposé automatique du tramway sur la fréquentation des transports collectifs (Bonnel, Cabanne et Massot, 2003), appuyées par la multiplication des approches psycho-sociologiques du choix modal (Kaufmann, 2001). Une comparaison des 14 agglomérations ayant fait le choix du tramway avec un panel d'agglomérations organisées autour de l'autobus doit permettre d'approfondir la controverse autour de "l'effet-tramway ». Enfin, plus de 20 années séparent Nantes de Nice, qui sont les mises en services la plus ancienne et la plus récente du panel. Il est possible d'introduire une dimension diachronique jusqu'alors absente des travaux d'évaluation: y a-t-il une évolution au fil du temps des effets du tramway sur la fréquentation des transports collectifs, et quels enseignements en tirer?

4 Afin de saisir dans leur totalité les enjeux du bilan mené ici, nous proposons dans un premier temps de présenter en détail les éléments de contexte qui fondent cette recherche : l'évolution de l'offre de tramway dans les agglomérations de province en France depuis plus de deux décennies, et la mise en question de l'efficacité du tramway sur la fréquentation par des travaux sur le choix modal. La méthodologie vise ensuite à répondre à la fois à la question de l'effet du tramway sur la fréquentation des transports publics, et à celle de leur évolution au fil du temps, à partir de l'exploitation des annuaires statistiques édités par le CERTU. Nous serons alors en mesure de proposer un bilan actualisé, portant successivement sur "l'effet tramway » et sur son évolution diachronique, avant d'interroger la place des facteurs en débat.

\section{Un bilan à actualiser}

5 Le travail mené ici se justifie doublement : par la multiplication des mises en services, qui conduit à un rappel historique de la réintroduction du tramway dans les agglomérations de province (1.1.), et par l'émergence d'un débat sur les effets réels de cette réintroduction sur la fréquentation des transports publics de province, entre bilan favorable et éléments de mise en question (1.2.).

\section{A. Une multiplication récente des mises en service de tramways dans les agglomérations de province}

6 Au milieu des années 1960, seules trois agglomérations conservent une ligne de leur réseau historique de tramway: Lille, Marseille et Saint-Étienne. Partout ailleurs, la politique du «tout voiture » conduit à démanteler les réseaux existants. Pourtant, au cours des années 1970, le choc pétrolier de 1973 et les limites de la politique en faveur de l'automobile conduisent les pouvoirs publics à envisager une réhabilitation des transports collectifs, et notamment du tramway: en 1975, le Secrétariat d'Etat aux Transports lance un concours auprès de huit agglomérations afin de réaliser les premières lignes (Zalkind, 2008). C'est cependant Nantes, qui ne faisait pas partie de ce concours, qui inaugure le premier réseau moderne de tramway, en 1985. Elle est suivie par Grenoble, deux ans plus tard. Cette impulsion locale s'inscrit dans le cadre nouveau 
de la LOTI², qui, votée en 1982, place les transports collectifs urbains sous l'autorité des communes et de leurs groupements.

7 Le temps de gestation des projets est plus long dans les autres agglomérations, puisque c'est à partir du milieu des années 1990 que se multiplient les réalisations, dans la lancée de Rouen et Strasbourg, qui inaugurent leurs tramways en 1994. À partir de ce moment se construit une rhétorique du tramway, où le rééquilibrage face à l'automobile n'est plus le seul argument. Le tramway reste certes un outil au service d'un meilleur partage modal au bénéfice du transport public, mais il devient également, pour les techniciens comme pour les élus, un outil d'aménagement urbain, au service d'un réaménagement des espaces traversés et d'une structuration de l'urbanisation, dans le cadre d'enjeux à la fois sociaux et environnementaux (Beaucire et Lebreton, 2000). Quelques voix s'élèvent, qui mettent en question la pertinence notamment financière et technique de ces choix (Carmona, 2001), mais elles ne parviennent pas à ralentir le succès de ce mode de transport (Tableau 1).

Tableau 1 - Agglomérations ayant mis en service un réseau de tramway entre 1985 et 2007

\begin{tabular}{|r|r|r|r|r|r|}
\hline Agglomérations & $\begin{array}{r}\text { Mise en } \\
\text { service }\end{array}$ & Longueur & Agglomérations & $\begin{array}{r}\text { Mise en } \\
\text { service }\end{array}$ & Longueur \\
\hline Nantes & 1985 & 5,8 & Caen & 2003 & 15,7 \\
Grenoble & 1987 & 9,2 & Bordeaux & 2004 & 12,5 \\
Rouen & 1995 & 14,5 & Clermont-Fd. & 2006 & 14,2 \\
Strasbourg & 1995 & 9,8 & Mulhouse & 2006 & 10,5 \\
Montpellier & 2000 & 15,2 & Valenciennes & 2006 & 9,5 \\
Orléans & 2001 & 18 & Le Mans & 2007 & 15,4 \\
Nancy & 2002 & 11,3 & Nice & 2007 & 8,7 \\
\hline
\end{tabular}

Réalisation : V. Gagnière d'après des données Certu, 2011

8 Les années 2000 constituent un tournant dans le nombre de mises en service : alors que seules quatre agglomérations ont un réseau de tramway en 1999, une nouvelle mise en service a lieu chaque année entre 2000 et 2004. Après une pause en 2005, le mouvement repart de plus belle avec trois mises en service en 2006, et deux en 2007. Ce sont au total 14 agglomérations (cf. Tableau 1), dont les périmètres de transport urbain rassemblent plus de 5 millions d'habitants, qui ont réintroduit un réseau de tramway au cours des 22 années étudiées. Mises à part les agglomérations millionnaires, celles ayant conservé leur réseau de tramway historique et celles organisées autour du métro (Rennes et Toulouse), ce sont 11 agglomérations de plus de 250000 habitants sur 17 qui ont fait le choix du tramway. Seules trois agglomérations sont en dessous de ce seuil, Caen, Le Mans et Mulhouse, sur un ensemble de 38 agglomérations entre 100000 et 250000 habitants (UTP, 2009). Cette tendance à la multiplication des réalisations a conduit à un fort développement des évaluations a posteriori, parmi lesquelles celle des effets de ces réintroductions sur la fréquentation du transport public. Elle engendre un débat qui justifie de renouveler l'évaluation de ces effets sur l'usage du transport collectif. 


\section{B. Faire émerger un débat pour une meilleure compréhension des effets du tramway sur la fréquentation des transports collectifs}

Plusieurs analyses s'attachent à mesurer l'efficacité du site propre introduit par le tramway. Nous nous fondons sur celle réalisée par Francis Beaucire en 2006. Ce travail s'appuie sur les six premières mises en service de tramways modernes, à Nantes, Grenoble, Rouen, Strasbourg, Montpellier et Orléans. Il propose une évaluation de l'impact de la réintroduction du tramway sur la fréquentation globale des transports collectifs, sans occulter le fait qu'une analyse du partage modal serait nécessaire pour interroger l'objectif de report modal depuis l'automobile. Francis Beaucire recourt au nombre de voyages par habitant, indicateur le plus global pour estimer l'usage d'un réseau de transport collectif. L'évolution de la fréquentation entre l'année qui précède la mise en service et les deux années qui lui succèdent s'établit de 26 à $46 \%$ pour les six agglomérations étudiées. En comparaison, les autres agglomérations connaissent une évolution entre -3 et $5 \%$. Ces chiffres permettent à Francis Beaucire de conclure que " nulle par en France, et depuis bien longtemps, des résultats aussi nets n'ont été obtenus. Les TCSP [transports collectifs en site propre], et plus particulièrement une seule ligne fortement structurante, entraînent des accroissements de l'ensemble des indicateurs [...]. Si l'on réfere ces résultats à la moyenne enregistrée par les villes de taille comparable qui n'ont pas investi dans une ligne de tramway en site propre, les résultats sont spectaculaires » (p. 19).

Expliquer ces résultats nécessite de prendre en considération un ensemble de données de contexte issues du transport, de l'urbanisation et des modes de vie, qui les interrogent à plusieurs titres. Tout d'abord, la hausse de la fréquentation dans les agglomérations ayant fait le choix du tramway est à mettre au regard de l'évolution de l'offre de transport public, tant quantitativement que qualitativement. Dans l'ensemble des agglomérations concernées, la hausse de la fréquentation est sans mesure par rapport à celle de l'offre : $+40 \%$ de fréquentation à Rouen ou Strasbourg deux ans après les mises en service (Bonnel, Cabanne et Massot, 2003, p. 18), contre des hausses d'offre respectivement de +1 et $+6 \%$ (Certu, 1990-2011). En outre, l'augmentation de fréquentation se situe sur les axes concernés par les sites propres, le reste de l'agglomération ne bénéficiant pas toujours des retombées positives de ces investissements, les gains de fréquentation liés au tramway masquant une baisse ailleurs (idem). Le débat porte donc sur le rapport entre les investissements consentis et les résultats observés, et sur la réalité géographique des hausses de fréquentation au sein des agglomérations.

11 En parallèle, il est nécessaire de replacer l'usage du transport public dans une approche multimodale, plus proche de la réalité et des objectifs annoncés. L'introduction d'un réseau de tramway est-elle suffisante pour réduire la part modale de l'automobile? Jusqu'au début des années 2000, la réponse est négative (Bonnel, Cabanne et Massot, 2003). En effet, si le réaménagement de voirie consécutif à l'implantation d'un réseau de tramway conduit à réduire la place de l'automobile, il s'accompagne, malgré la création de parkings relais en périphérie, de mesures favorables à l'automobile dans les centres urbains, telles que la suppression du transit automobile par la réalisation de rocades, ou la multiplication des capacités de stationnement (Frenay, 2004). Dans cette perspective, donc, l'efficacité réelle de la politique en faveur du tramway doit être analysée au regard de l'évolution de l'usage de l'automobile. 
12 Enfin, l'argumentation précédente relève d'une approche traditionnelle du choix modal, qui fait de l'individu un être rationnel choisissant un mode de transport par la minimisation de ses coûts monétaires et temporels. De nombreux travaux insistent sur l'importance de facteurs socio-psychologiques : le choix modal se construit également sur la perception subjective des différents modes de transport, et sur la force des habitudes modales (Kaufmann, 2001). Ces deux pistes ont été creusées récemment, les travaux tendant à montrer que le changement des habitudes de déplacement se heurte à la remise en cause des routines, nécessitant un élément déclencheur fort dans la vie des individus (Rocci, 2007 ; Vincent, 2008). Dans le cas qui nous concerne, ces résultats interrogent donc à la fois les motivations des individus et la pérennité de leur choix modal en faveur du tramway : l'introduction de ce mode de transport constitue-t-elle à elle seule un facteur à même de modifier durablement les habitudes de transport des individus?

13 Permise par le doublement du panel, la nouvelle évaluation que nous proposons de l'effet du tramway sur la fréquentation du transport public fait donc face à de nouveaux enjeux concernant son contexte modal, notamment vis-à-vis de la voiture, et les motivations socio-psychologiques qui régissent les choix modaux. Sans les occulter, cet article s'attache avant tout à mettre à jour le bilan de l'impact du tramway sur la fréquentation du transport public, dont nous présentons maintenant la méthodologie.

\section{Méthode d'analyse}

14 Nous abordons d'abord les grandes lignes de la méthodologie développée ici (A.), avant de présenter en détail le choix du panel d'agglomérations témoins, qui permet d'analyser la réalité de l'effet du tramway (B.), et d'introduire l'approche diachronique dans l'analyse de cet effet (C.).

\section{A. Grandes lignes méthodologiques : choix du panel, sources et indicateurs, temporalités d'analyse}

15 Le panel est construit de façon restrictive. Cherchant à cerner l'effet de la réintroduction du tramway sur la fréquentation des transports publics, deux types d'agglomérations ont été exclues : celles qui n'ont pas cessé d'exploiter un réseau de tramway - Lille, Marseille et Saint-Etienne - car l'effet d'introduction y est absent, et celles qui, bien qu'ayant mis en service un réseau de tramway, sont organisées autour d'un transport collectif plus lourd de type métro, comme Lyon. D'un point de vue technique, le terme " tramway » est entendu dans une acception large, intégrant aussi bien les modes sur fer, qui constituent la majorité des cas, que sur pneus, exploités à Nancy, Caen et Clermont-Ferrand ; les deux premières agglomérations ont été touchées par d'importants problèmes techniques nuisant à la fréquentation.

Les données utilisées sont issues des annuaires statistiques édités par le CERTU sur 196 réseaux de transports urbains en France. Ces données proviennent directement des autorités organisatrices et sont publiées en l'état, si bien qu'il est possible de s'interroger sur leur fiabilité, pour une même agglomération d'une année sur l'autre, et pour la comparaison entre plusieurs agglomérations. Néanmoins, c'est la seule base de données qui offre un panel d'indicateurs couvrant l'ensemble des mises en services modernes de tramway à partir de 1985. L'indicateur disponible est le nombre de 
voyages par habitant, soit le nombre moyen de trajets effectués chaque année par un habitant sur un mode de transport. Un déplacement effectué avec deux modes différents équivaut donc à deux voyages.

17 Enfin, les périodes de temps retenues pour évaluer l'effet de la mise en service du tramway sur la fréquentation du transport public diffèrent de l'analyse réalisée en 2006. Le temps d'évolution de la fréquentation avant la mise en service est étendu à 3 ans : ne prendre en compte que l'année précédente présente un biais dans la mesure où les travaux du tramway entraînent une modification de la desserte en transport public, et conduisent à une diminution artificielle de la fréquentation. Étendre cette période à trois ans permet de limiter ce biais. En ce qui concerne la période suivant la mise en service, elle est également étendue à trois ans, afin de prendre en compte dans toute sa durée le temps de montée en charge. Au total, la période étudiée court donc de 1982 à 2009, dernière année de disponibilité de l'indicateur.

\section{B. Le choix du panel d'agglomérations témoins}

18 Ce panel est nécessaire pour individualiser l'effet de la réintroduction d'un réseau de tramway sur la fréquentation du transport public. Il est constitué par un ensemble d'agglomérations sans réseau de tramway ni réseau plus lourd de type métro, donc organisé autour de l'autobus. Traditionnellement, ce panel est constitué d'agglomérations réparties en grandes catégories en fonction de la population du périmètre de transport urbain. Or, cette méthode a pour conséquence de séparer dans deux catégories distinctes des agglomérations dont la population est proche. Par conséquent, le choix est fait ici d'individualiser le panel à plus ou moins $25 \%$ de la population de l'agglomération étudiée. Ainsi, pour une agglomération de 200000 habitants, le panel témoin comprend l'ensemble des agglomérations dont la population est comprise entre 150000 et 250000 habitants. La diminution au fil du temps de la taille des agglomérations mettant en service une ligne de tramway permet de conserver un panel suffisant d'agglomérations témoins, ce qui compense la multiplication des mises en service. Afin d'obtenir un échantillon représentatif, un minimum de cinq agglomérations est nécessaire pour constituer le panel. Si l'écart de $25 \%$ n'est pas suffisant, les agglomérations suivantes en termes de population sont sollicitées, jusqu'à ce que le panel soit constitué.

\section{Le choix d'un découpage diachronique}

19 La spécificité de notre bilan par rapport à celui de 2006 est de proposer une approche diachronique de l'effet du tramway sur la fréquentation du transport public. Pour cela, il est nécessaire de proposer un découpage temporel. Dans la mesure où cet article étudie l'effet du tramway sur la fréquentation du transport public par rapport à un panel d'agglomérations, c'est cette fréquentation moyenne de l'ensemble des agglomérations étudiées qui sert de base au découpage diachronique: elle est révélatrice de l'évolution globale de l'usage du transport public, et permet d'en individualiser un hypothétique effet tramway (Figure 1). 
Figure 1. Évolution moyenne de la fréquentation des transports publics dans les agglomérations françaises de province entre 1982 et 2009

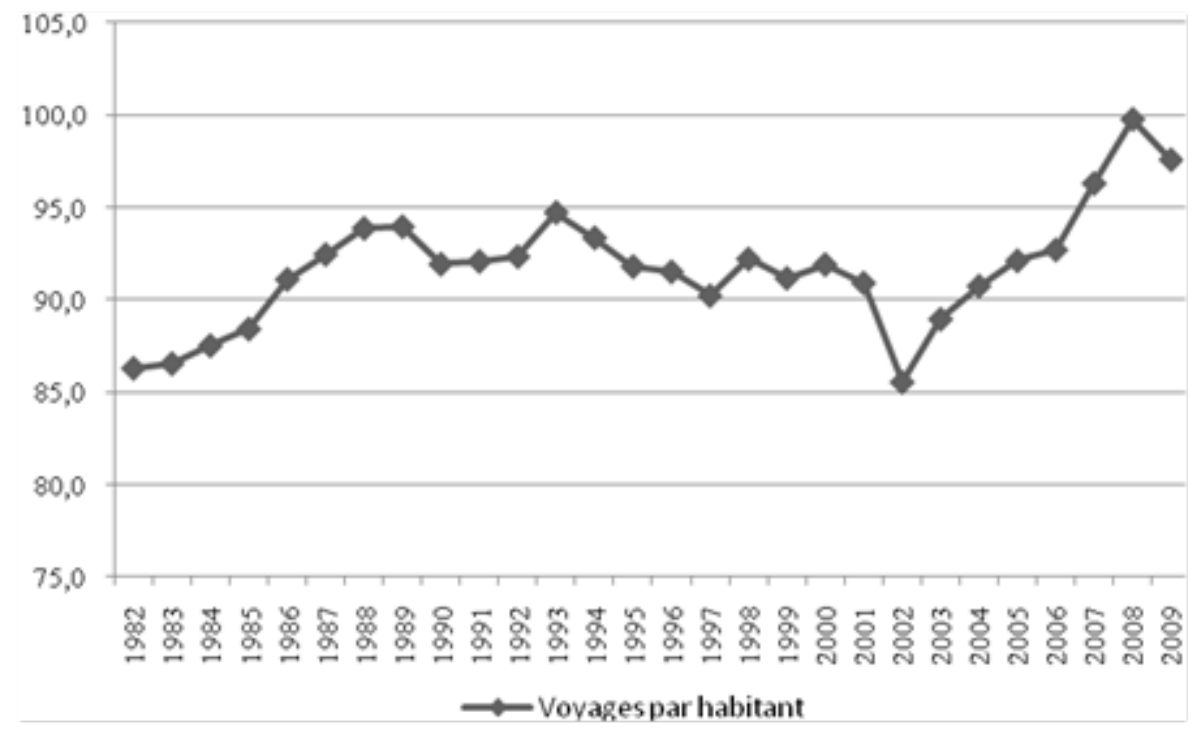

Trois périodes peuvent être individualisées : une hausse continue de 1982 à 1989, suivie d'une période plus chaotique orientée à la baisse entre 1990 et 2002. Enfin, à partir de 2003, la remontée de fréquentation du transport public est très nette. Les mises en service de tramway se trouvent réparties comme indiqué dans le Tableau 2. Cette périodisation constitue la base de l'interrogation sur un potentiel effet temporel dans l'impact de la mise en service du tramway sur la fréquentation du transport public.

Tableau 2 - Mises en service de tramway entre 1985 et 2007 en fonction du découpage diachronique de la fréquentation moyenne du transport public

\begin{tabular}{|l|l|l|}
\hline \multicolumn{1}{|c|}{$\begin{array}{c}\text { 1982-1989 : hausse } \\
\text { modérée }\end{array}$} & \multicolumn{1}{|c|}{$\begin{array}{c}\text { 1990-2002 : baisse } \\
\text { irrégulière }\end{array}$} & \multicolumn{1}{|c|}{$\begin{array}{c}\text { 2003-2009 : hausse } \\
\text { forte }\end{array}$} \\
\hline Nantes (1985) & Rouen (1995) & Caen (2003) \\
Grenoble (1987) & Strasbourg (1994) & Bordeaux (2004) \\
& Montpellier (2000) & Clermont-Fd. (2006) \\
& Orléans (2001) & Mulhouse (2006) \\
Nancy (2002) & $\begin{array}{l}\text { Valenciennes (2006) } \\
\text { Le Mans (2007) } \\
\text { Nice (2007) }\end{array}$ \\
& & \\
\hline
\end{tabular}

\section{Bilan actualisé}

Ce bilan s'organise en trois temps: il individualise d'abord l'effet du tramway en comparant les agglomérations ayant fait ce choix à celles qui restent organisées autour d'un réseau de bus (3.1.). Il interroge ensuite la validité d'un découpage diachronique (3.2.), avant de proposer des pistes explicatives liées aux enjeux modaux et sociopsychologiques déterminés plus haut (3.3.). 


\section{A. Un effet tramway confirmé par la comparaison avec un panel témoin}

22 Le Tableau 3 constitue la base de l'analyse : il présente l'évolution de la fréquentation du transport public avant et après la mise en service du tramway dans les 14 agglomérations concernées, et compare ces chiffres à un panel d'agglomérations témoins.

Tableau 3. Évolution de la fréquentation du transport public avant et après la mise en service du tramway ; comparaison avec un panel d'agglomérations témoins

\begin{tabular}{|l|r|r|r|r|}
\hline & \multicolumn{2}{|c|}{ Agglomération } & \multicolumn{2}{c|}{ Panel témoin } \\
\hline & A-3 à A-1 & A0 à A+3 & A-3 à A-1 & A0 à A+3 \\
\hline Nantes & $4,8 \%$ & $7,3 \%$ & $1,3 \%$ & $1,0 \%$ \\
\hline Grenoble & $-5,8 \%$ & $27,3 \%$ & $1,7 \%$ & $1,2 \%$ \\
\hline Rouen & $11,6 \%$ & $10,1 \%$ & $-3,0 \%$ & $-1,2 \%$ \\
\hline Strasbourg & $-2,3 \%$ & $8,9 \%$ & $-1,8 \%$ & $3,5 \%$ \\
\hline Montpellier & $5,6 \%$ & $3,8 \%$ & $-1,1 \%$ & $-9,7 \%$ \\
\hline Orléans & $-5,5 \%$ & $25,6 \%$ & $-1,0 \%$ & $-1,6 \%$ \\
\hline Nancy & $-17,6 \%$ & $23,7 \%$ & $0,0 \%$ & $0,3 \%$ \\
\hline Caen & $3,2 \%$ & $9,6 \%$ & $-9,8 \%$ & $-0,1 \%$ \\
\hline Bordeaux & $-11,2 \%$ & $37,9 \%$ & $-15,9 \%$ & $11,3 \%$ \\
\hline Clermont-Fd. & $-5,2 \%$ & $37,3 \%$ & $4,7 \%$ & $-0,3 \%$ \\
\hline Moulhouse & $-34,2 \%$ & $11,2 \%$ & $4,2 \%$ & $-0,2 \%$ \\
\hline Valenciennes & $-3,5 \%$ & $8,1 \%$ & $2,8 \%$ & $0,7 \%$ \\
\hline Le Mans* & $-3,5 \%$ & $14,2 \%$ & $-3,6 \%$ & $-2,3 \%$ \\
\hline Nice* & $131,4 \%$ & $30,8 \%$ & $2,6 \%$ & $-1,7 \%$ \\
\hline Moyenne & $4,8 \%$ & $18,3 \%$ & $-1,3 \%$ & $0,1 \%$ \\
\hline
\end{tabular}

* Pour Le Mans et Nice, seules les deux années suivant la mise en service sont disponibles. Réalisation : V. Gagnière d'après des données Certu

23 Si l'on s'intéresse à la moyenne des résultats, qui constitue la donnée la plus agrégée, l'effet de la mise en service du tramway sur la fréquentation des transports publics est 
indéniable, aussi bien pour les réseaux concernés, pour lesquels l'évolution de la fréquentation croît d'un facteur quatre - de 4,8 \% à 18,3\% -, qu'en comparaison avec la moyenne des panels témoins, où la fréquentation est stable. Le cas niçois fait cependant exception, et sera analysé au paragraphe suivant. Sans celui-ci, la fréquentation avant mise en service est de $-4,9 \%$. Ceci peut être dû aux travaux consécutifs aux ouvertures de lignes, bien qu'il ne soit pas possible d'en déterminer l'influence précise. Au final, le doublement du panel n'a cependant pas modifié fondamentalement les conclusions du bilan mené en 2006 par Francis Beaucire. Il aurait été intéressant de comparer les données actualisées avec les résultats précédents, mais le choix d'une période d'analyse différente ne le permet pas.

Une analyse de détail révèle toutefois de fortes disparités. Bien que l'effet du tramway soit positif dans les 14 agglomérations, deux éléments de divergence méritent d'être soulignés. D'un côté, l'effet direct du tramway sur la fréquentation trois ans après la mise en service est très variable : celle-ci augmente de 3,8 \% à Montpellier à $37,9 \%$ à Bordeaux, soit un facteur de un à dix. Le contexte local a une influence sur les résultats: Bordeaux et Orléans sont deux des agglomérations où le trafic a le plus crû, alors qu'une seconde ligne de tramway y était mise en service dans les trois ans suivant l'ouverture de la première; à l'inverse, il ne semble pas exister de relation entre la longueur de la ligne et l'évolution de la fréquentation (cf. Tableau 1). Ces disparités au sein du panel sont également visibles dans l'évolution avant/après la mise en service du tramway. Neuf agglomérations connaissent une baisse de leur fréquentation avant la mise en service, probablement en conséquence de la phase de travaux. Suite à la mise en service, trois agglomérations voient leur fréquentation évoluer plus faiblement après qu'avant. La différence est faible à Rouen, et de deux points à Montpellier. La troisième agglomération concernée est Nice, dont le cas est spécifique: la fréquentation progresse de 131,4\% avant la mise en service du tramway, ce qui est lié à une refonte complète du réseau de transport urbain, consécutivement à la création de la communauté urbaine en 2005.

Les disparités locales sont donc fortes, eu égard au contexte spécifique de chaque agglomération. Les principales divergences sont relatives à l'intensité de l'effet de l'introduction du tramway sur la fréquentation du transport public, mais elles ne remettent nullement en cause la tendance générale : la mise en service d'une ligne de tramway a bien un effet positif, à des degrés divers, sur la fréquentation du transport public. Un premier aperçu a été donné d'une possible évolution diachronique de cet effet, ce qui nous conduit à aborder cette deuxième partie de l'analyse.

\section{B. Un effet diachronique calqué sur la fréquentation globale du transport public}

26 À partir de l'analyse de l'évolution de la fréquentation globale, trois périodes ont été distinguées : une faible hausse entre 1982 et 1989, une baisse chaotique entre 1990 et 2002, et une forte hausse entre 2003 et 2009. Deux objets sont à comparer entre ces trois séries : l'évolution de la fréquentation du transport public dans les agglomérations ayant mis en service un tramway, et la différence d'évolution entre ces agglomérations et les panels témoins. La Figure 2 offre une vision globale de ces indicateurs. 
Figure 2. Évolution de la fréquentation du transport public (en voyages annuels par habitant) selon un découpage en trois périodes pour les agglomérations ayant mis en service une ligne de tramway (A étant l'année de mise en service) et les agglomérations témoins

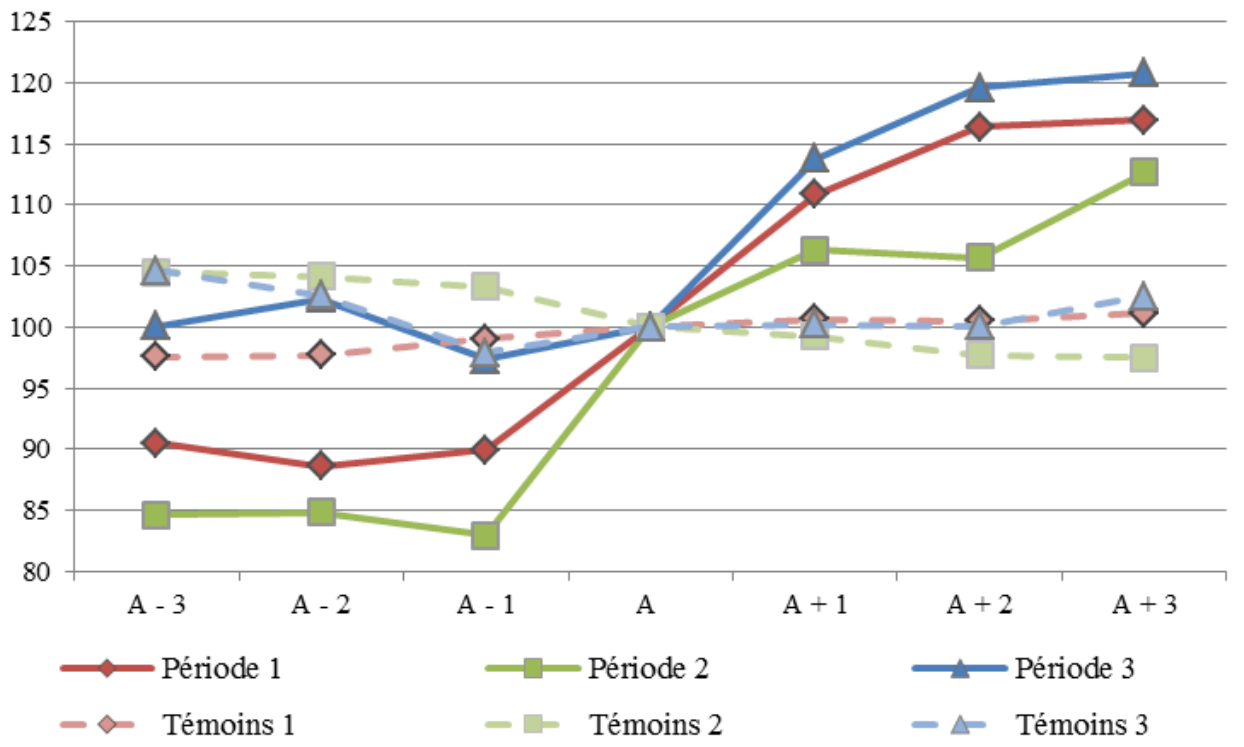

Réalisation : V. Gagnière d'après des données Certu

La comparaison de l'effet de la mise en service du tramway au cours des trois périodes retenues révèle un différentiel : la hausse est plus nette au cours de la période récente (2003-2009), suivie de la période la plus ancienne (1982-1989). Dans ces deux cas, elle prend une forme parabolique, la progression s'atténuant d'année en année. La période centrale (1990-2002) connaît une évolution plus faible, et plus chaotique. Ces tendances sont à mettre en relation avec celles des agglomérations témoins, puisque leur fréquentation évolue selon des tendances similaires, quoique bien plus faibles : la plus forte évolution de fréquentation après l'année $\mathrm{A}$ a lieu au cours de la période la plus récente, suivie de la plus ancienne, tandis que la période intermédiaire connait un phénomène de baisse. L'analyse des évolutions présente ces résultats sous une autre forme (Tableau 4).

Tableau 4. Évolution de la fréquentation des transports publics par période les trois années suivant la mise en service d'une ligne de tramway, et dans un panel d'agglomérations témoins

\begin{tabular}{|l|r|r|}
\hline & Agglomérations & Panel témoin \\
\hline Période 1 & $16,9 \%$ & $1,1 \%$ \\
\hline Période 2 & $12,7 \%$ & $-2,5 \%$ \\
\hline Période 3 & $20,8 \%$ & $2,5 \%$ \\
\hline
\end{tabular}

Réalisation : V. Gagnière d'après des données Certu

Cette interrogation sur l'existence d'une influence diachronique sur l'effet tramway vient donc compléter l'actualisation du bilan réalisé par Francis Beaucire en 2006. L'effet du tramway sur la fréquentation du transport public diffère, de quatre points en 
moyenne, selon la période étudiée. En parallèle, l'évolution de l'usage des transports collectifs dans le panel témoin connaît une tendance identique. La mise en service d'une ligne de tramway a donc non seulement un impact fort sur la fréquentation du transport public, mais cet impact est lui-même fonction de la conjoncture du transport public. En d'autres termes, le tramway a un effet indéniable sur la fréquentation, mais n'échappe pas aux variations du contexte socio-économique qui influence celle-ci. Cette conclusion justifie d'autant plus l'interrogation sur les pistes explicatives à apporter aux résultats.

\section{Des facteurs explicatifs ouvrant des pistes de recherche pour affiner le bilan}

Le débat sur la réalité du succès du tramway sur la fréquentation du transport public a été ouvert dans trois directions. La première concerne une différenciation géographique de l'évolution de la fréquentation, entre une forte hausse dans les corridors desservis, et une baisse ailleurs. Cette approche nécessite une connaissance fine des pratiques de mobilité des individus qui n'est rendue possible, dans les agglomérations de province, qu'à partir des données des Enquêtes Ménages Déplacements (EMD), ce qui n'est pas l'objet de cet article. Elle soulève néanmoins une question de fond. Le choix fait du tracé des lignes s'accompagne d'une refonte globale du réseau de transport, et d'un rabattement généralisé des lignes de bus sur la ou les ligne(s) de tramway. Dans ces conditions, il est possible que la hausse du nombre de voyages soit artificiellement gonflée par la hausse contrainte du nombre de correspondances. Le moyen de le vérifier est de comparer le nombre de voyages au nombre de déplacements effectués, ceux-ci ne prenant pas en compte les correspondances. Encore une fois, une analyse des EMD est nécessaire, mais celles-ci ne sont disponibles que de manière ponctuelle, ce qui limite leur pertinence. Il n'empêche que ce type de recherche permettrait de connaître l'impact réel du tramway sur la fréquentation des transports publics.

La seconde direction évoquée consistait à replacer le tramway dans une perspective multimodale, en s'interrogeant sur ses effets sur l'usage de l'automobile. Bien que le report modal depuis la voiture particulière ne soit nullement automatique, la comparaison des parts modales de ce mode de transport et du transport public suite à l'ouverture d'une ligne de tramway constitue un premier élément de réponse. Au regard de la disponibilité des EMD, cinq agglomérations peuvent être analysées: Nantes, Grenoble, Strasbourg, Bordeaux et Nice (Tableau 5).

Tableau 5. Évolution de la part modale de l'automobile et du transport public au regard de la mise en service d'une ligne de tramway

\begin{tabular}{|l|l|r|r|r|r|}
\hline & & \multicolumn{2}{|c|}{$\begin{array}{c}\text { Part modale de } \\
\text { I'automobile }\end{array}$} & \multicolumn{2}{c|}{$\begin{array}{c}\text { Part modale du } \\
\text { transport public }\end{array}$} \\
\hline & $\begin{array}{l}\text { Dates des } \\
\text { EMD }\end{array}$ & $1^{\text {ère EMD }}$ & $2^{\text {nde EMD }}$ & $1^{\text {ère EMD }}$ & $2^{\text {nde } E M D}$ \\
\hline Nantes (1985) & $1980-1990$ & $45 \%$ & $59 \%$ & $14 \%$ & $13 \%$ \\
\hline
\end{tabular}




\begin{tabular}{|l|l|r|r|r|r|}
\hline $\begin{array}{l}\text { Grenoble } \\
(1987)\end{array}$ & $1985-1992$ & $48 \%$ & $54 \%$ & $10 \%$ & $14 \%$ \\
\hline $\begin{array}{l}\text { Strasbourg } \\
(1995)\end{array}$ & $1988-1997$ & $49 \%$ & $52 \%$ & $7 \%$ & $9 \%$ \\
\hline $\begin{array}{l}\text { Bordeaux } \\
(2004)\end{array}$ & $1998-2009$ & $67 \%$ & $64 \%$ & $8 \%$ & $9 \%$ \\
\hline Nice (2007) & $1998-2009$ & $57 \%$ & $53 \%$ & $6 \%$ & $8 \%$ \\
\hline
\end{tabular}

Réalisation : V. Gagnière, d'après des données Certu, 2011

31 À Nantes, Grenoble et Strasbourg, la part modale de l'automobile augmente malgré la mise en service des premières lignes de tramway, celles-ci ayant eu lieu au cours de décennies très favorables à la voiture particulière (Guidez, 2002). Nous rejoignons ici les analyses portées par Patrick Bonnel, Isabelle Cabanne et Marie-Hélène Massot: l'évolution à la fois quantitative et qualitative de l'offre consécutive à la mise en service d'un tramway ne parvient pas toujours à relancer la part modale du transport public, et n'a surtout aucun effet sur celle de l'automobile, qui continue de croitre, la mobilité quotidienne n'évoluant pas à somme constante. La situation est différente à Bordeaux et à Nice, agglomérations marquées par une forte congestion routière, où l'époque d'introduction du tramway est concomitante d'une baisse de la part modale de la voiture particulière. Toutefois, il n'existe pas forcément de lien direct: les baisses de part modale de l'automobile ne sont pas mécaniquement compensées par les hausses en transport public. À Nice, l'usage de la voiture particulière diminue de 0,40 déplacement quotidien par habitant, la mobilité globale de 0,48 , le transport public en gagnant 0,03 , ce qui ne suffit pas à parler de compensation. À Bordeaux, si l'usage de l'automobile diminue de 0,07 déplacement quotidien par habitant, la mobilité globale crô̂t de 0,10, de façon répartie entre le vélo $(+0,02)$, le transport public $(+0,05)$, et surtout la marche à pied $(+0,07)$. Dans les deux cas, l'introduction d'un tramway semble donc n'avoir qu'un rôle limité, si ce n'est nul, sur l'usage de la voiture particulière, ce qui a été démontré sur d'autres terrains (Prud'homme, Koning, Kopp, 2008). La question de la diminution d'usage de l'automobile, et de ses effets sur la mobilité globale reste donc en suspens. La littérature actuelle tend à rapprocher cette évolution de facteurs tels que la hausse du prix du pétrole et la faible croissance économique, que ce soit à l'échelle des agglomérations par l'analyse des EMD les plus récentes (Certu, 2007 ; Quételard, 2008), ou à l'échelle nationale (Paul-Dubois-Taine, 2007).

Enfin, la troisième direction ouverte s'orientait vers une analyse socio-psychologique du choix modal. Cette approche permettrait de s'interroger sur les raisons du processus de transfert modal au profit du tramway chez les individus, et notamment sur la pérennité de ce transfert. À notre connaissance, ce type d'approche n'a pas été appliqué au tramway: les principaux travaux concernent les modes doux (Rocci, 2007), et les usages alternatifs de la voiture particulière, autopartage et covoiturage (Vincent, 2008). $\mathrm{Au}$ carrefour de la sociologie et de l'aménagement, il existe donc un champ plus qualitatif à défricher, qui permettrait de contribuer à la compréhension de l'attachement des individus au transfert modal opéré en direction du tramway. 


\section{Conclusion}

Cette étude portait sur 14 agglomérations qui, entre 1985 et 2007, ont mis en service une ou plusieurs lignes de tramway sur leur territoire. Angers et Reims les ont rejoints en 2011, et les travaux sont en cours dans cinq autres agglomérations, Besançon, Brest, Dijon, Le Havre et Tours, pour des mises en service s'échelonnant entre 2012 et 2015. La success story ne se dément donc pas. Du strict point de vue de ses effets sur la fréquentation, ce choix est justifié. La mise à jour d'un premier bilan réalisé par Francis Beaucire en 2006 confirme, sur les trois années suivant la mise en service, des gains de fréquentation de 4 à $40 \%$, là où, dans le même temps, les agglomérations comparées sont en situation moyenne de stagnation. L'introduction d'une approche diachronique a permis de compléter cette analyse, en soulignant que la fréquentation consécutive à la mise en service d'un tramway restait soumise aux aléas de la conjoncture du transport public, quoique dans des proportions très faibles.

Le débat peut alors être ouvert de deux façons. Il est tout d'abord possible d'interroger les causes intrinsèques de ce succès. Se pose alors la question à la fois des tracés de lignes, et de la refonte globale du réseau de transport, qui peut conduire à gonfler artificiellement la fréquentation. Le débat peut également s'élargir à une approche touchant au report modal : le report depuis l'automobile semble limité, tandis qu'un interrogation sur les ressorts socio-psychologiques du choix du tramway pourrait en éclairer les raisons et la pérennité. De façon générale, il faut souligner que la mobilité quotidienne n'évolue pas à somme constante, et donc que l'effet du tramway peut être évalué de façon fort différente selon que l'on s'interroge uniquement sur ses conséquences sur la fréquentation du transport public, ou également sur l'usage de la voiture particulière.

Enfin, rappelons que cette approche par la fréquentation ne constitue qu'une facette de l'évaluation a posteriori des politiques locales de transport en faveur des transports collectifs en site propre. Il est nécessaire de la replacer au sein de l'ensemble des approches existantes pour qui veut produire un bilan exhaustif des effets de la réintroduction du tramway dans les centres urbains en France depuis près de trois décennies.

\section{BIBLIOGRAPHIE}

Beaucire F., 2006, Transports collectifs urbains : quelle contribution au développement urbain durable et par quels moyens ?, Paris, Institut Veolia Environnement, 49 p.

Beaucire F., Lebreton J., 2000, Transports publics et gouvernance urbaine, Paris, Milan, 127 p.

Bonnel P., Cabanne I., Massot M.-H., 2003, Évolution de l'usage des transports collectifs et politiques de déplacements urbains, Paris, la Documentation française, $81 \mathrm{p}$.

Carmona M., 2001, Tramway, le coût d'une mode, Orléans, Paradigme, 200 p. 
Certu, 1990-2011, Annuaire statistique. Transports collectifs urbains, Lyon, Certu, 591 p.

Certu, 2007, La mobilité urbaine des années 2000. Vers un « découplage » entre la possession et l'usage de la voiture ?, Lyon, Certu, 8 p.

Certu,2011, EMD : tableaux des résultats, www.certu.fr, mis en ligne le 13 avril 2011, consulté le 18 août 2011.

Frenay P., 2004, « Le tram, outil au service du développement durable? », TEC, Volume 184, p. 62-69.

Guidez J.-M., 2002, La mobilité urbaine en France : les années 1990, Lyon, Certu, 103 p.

Kaufmann V., 2001, Mobilité quotidienne et dynamiques urbaines. La question du report modal, Lausanne, Presses polytechniques et universitaires romandes, $252 \mathrm{p}$.

Mercier A., 2008, Accessibilité et évaluation des politiques de transport en milieu urbain : le cas du tramway strasbourgeois, Lyon, Université Lumière Lyon 2, 310 p.

Paul-Dubois-Taine O., 2007, « Stagnation de la circulation automobile en France depuis cinq ans : accident conjoncturel ou inflexion durable? », Infrastructures et mobilité, Volume 72, p.8-10.

Prud'homme R., Koning M., Kopp P., 2008, « Paris, un tramway nommé désir », Transports, Volume 447, p.28-39.

Prud'homme R., Koning M., Kopp P., 2011, « Substituing a tramway to a bus line in Paris: costs and benefits », Transport Policy, Volume 18, $\mathrm{N}^{\circ} 4$, p.563-572.

Quételard B., 2008, « Du nouveau dans le « partage modal »? ", Transports urbains, Volume 112, p. 9-12.

Rocci A., 2007, De l'automobile à la multimodalité ? Analyse sociologique des freins et leviers au changement de comportements vers une réduction de l'usage de la voiture. Le cas de la région parisienne et perspective internationale, Paris, Université Paris 5 René Descartes, 542 p.

Stambouli J., 2007, « Les territoires du tramway moderne : de la ligne à la ville durable », Développement durable et territoires, mis en ligne le 11 juin 2007, consulté le 17 août 2011.

UTP, 2009, Les chiffres clés du transport public urbain 2008, Paris, UTP, 38 p.

Vincent S., 2008, Les « altermobilités » : analyse sociologique d'usages de déplacements alternatifs à la voiture individuelle. Des pratiques en émergence ?, Paris, Université Paris 5 René Descartes, $417 \mathrm{p}$.

Zalkind S., 2008, «Le renouveau du tramway », Revue générale des chemins de fer, Volume 170, p.7-10.

\section{NOTES}

1. Centre d'études sur les Réseaux, les Transports, I'Urbanisme et les constructions.

2. Loi d'Orientation sur les Transports Intérieurs. 


\title{
RÉSUMÉS
}

Cet article propose d'actualiser un bilan réalisé en 2006 des effets de la réintroduction du tramway sur la fréquentation du transport public dans les agglomérations françaises de province. Sa démarche se légitime par la multiplication des mises en service, permettant de doubler le panel, et par l'ouverture d'un débat sur les effets réels du tramway, mis en question par des approches empiriques et socio-psychologiques. L'analyse se construit à partir de la fréquentation du transport public publiée annuellement dans les annuaires statistiques du CERTU ${ }^{1}$. Se fondant sur les mises en service de tramway entre 1985 et 2007, elle s'interroge à la fois sur ses effets sur la fréquentation du transport collectif, et sur celle de leur évolution diachronique au cours des deux décennies étudiées. Les résultats confirment que l'introduction du tramway a un effet fort sur la fréquentation, quoiqu'elle soit légèrement sensible aux aléas conjoncturels. Néanmoins, ses effets semblent minimes en termes de report modal depuis l'automobile, tandis que deux points méritent d'être approfondis pour une meilleure compréhension: le rôle dans ce succès de la refonte du réseau de transport, et les motivations socio-psychologiques à l'origine du choix modal du tramway.

This article aims to update the 2006 assessment of the tram reintroduction on public transport use in the provincial French urban areas. It is justified by growing implementations, which double the sample group, and by the opening of a debate on real tram effects on public transport use, which are questioned by empirical and socio-psychological approaches. The analysis is built on public transport use published in annual statistical yearbooks edited by the CERTU. It is based on tram implementations between 1985 and 2007, and it questions both the reality of the reintroduction effects on public transport use, and the reality of a diachronic evolution within the two decades. Results tend to confirm the strong effect of tram implementation on public transport use, though slightly sensitive to socio-economic hazards. However, its effects on modal shift from the car seem low, and two points need to be stressed out for a better understanding: the role in the tram success of the transport network overhaul, and the socio-psychological motives regarding tram modal choice.

\section{INDEX}

Mots-clés : tramway, fréquentation du transport public, France, agglomérations de province

Keywords : tram, public transport use, France, provincial urban areas

\author{
AUTEUR \\ VINCENT GAGNIÈRE \\ Doctorant. Université Paris 1 Panthéon-Sorbonne / UMR Géographie-cités, équipe CRIA. \\ vincent.gagniere[chez]gmail.com
}

